\title{
Health related quality of life of gynaecologic cancer patients attending at Tikur Anbesa Specialized Hospital (TASH), Addis Ababa, Ethiopia
}

Birhanu Abera Ayana', Shiferaw Negash², Lukman Yusuf², Wendemagegnhu Tigeneh ${ }^{3}$ and Demewoz Haile ${ }^{4^{*}}$ (D)

\begin{abstract}
Background: Being diagnosed with gynaecologic malignancy certainly will have different sequelae which can hamper quality of life (QOL).This study aimed to assess health related quality of life (HRQOL) among gynaecologic cancer patients attending at Tikur Anbesa Specialized Hospital (TASH), Addis Ababa, Ethiopia.

Methods: This study employed facility-based cross-sectional study design on 153 gynaecological cancer patients attending TASH using the Amharic version of the European Organization for Research and Treatment of Cancer Quality of Life Core Questionnaire (EORTC QLQ-C30). We used descriptive statistics, independent t test and one way analysis of variance (ANOVA) in statistical analysis.

Results: The mean Global Health Status (GHS) was 40.95(SD \pm 24.35 ) and of the functional scores, social function was most affected (42.26, SD \pm 32.08 ), whereas cognitive function is the least affected domain (mean $=88.21, S D \pm 18.49$ ). The highest score on the symptom scores was found to be financial difficulties (mean $=64.76, \mathrm{SD} \pm 32.43$ ) followed by pain (mean $=55.12, S D \pm 29.64$ ) and fatigue (mean $=53.97, S D \pm 28.54)$; the lowest score on the contrary was scored for diarrhea (mean $=1.19, \mathrm{SD} \pm 7.38$ ). As stage increases there was a statistically significant reduction in GHS $(p=0.005)$ and in all functional score domains except the physical and emotional function. Advancement in stage of the disease has also affected significantly the symptom score domains except financial difficulties, nausea /vomiting and diarrhea. Patients who never went to school have scored a statistically significant lower score in GHS, physical function, role function and social function $(p<0.05)$.

Conclusion: GHS, social function, financial difficulties, pain and fatigue were the most affected domains; however, cognitive function and diarrhea were less affected components of HRQOL of gynaecologic cancer patients. Place of residence, educational status, marital status, payment type, cancer type and stage of the disease were associated with different quality of life scores.
\end{abstract}

Keywords: Gynaecologic cancer, Quality of life, EORTC QLQ-C30, Ethiopia

\footnotetext{
* Correspondence: demewozhaile@yahoo.com

${ }^{4}$ Department of Reproductive Health and Health Service Management,

School of Public Health, College of Health sciences, Addis Ababa University,

Addis Ababa, Ethiopia

Full list of author information is available at the end of the article
}

(c) The Author(s). 2018 Open Access This article is distributed under the terms of the Creative Commons Attribution 4.0 International License (http://creativecommons.org/licenses/by/4.0/), which permits unrestricted use, distribution, and reproduction in any medium, provided you give appropriate credit to the original author(s) and the source, provide a link to the Creative Commons license, and indicate if changes were made. The Creative Commons Public Domain Dedication waiver (http://creativecommons.org/publicdomain/zero/1.0/) applies to the data made available in this article, unless otherwise stated. 


\section{Background}

Quality of life (QOL) is a new dimension of care which has received greater attention in the last three decades. QOL is a multidimensional concept which is defined as a person's view of life, and with her satisfaction and pleasure with life [1]. QOL is concerned with social, emotional and physical well-being of patients following treatment, mirroring the World Health Organization's (WHO'S) definition of health [2].

Being diagnosed with gynaecologic malignancy certainly will have different sequelae which can hamper QOL. The special thing for gynaecological cancers is its effect on reproductive performance, sexuality and body image [3]. Understanding these impacts is vital to improve approaches to care, modify therapies and provide supportive care for the duration of the illness and enhance QOL.

Assessment of QOL among gynaecologic cancer patients provides supplementary information for physicians for selecting antineoplastic and supportive-care therapy. Managing QOL in gynaecologic cancer patients requires careful consideration of a variety of issues, many of which revolve around the surgical procedures employed and major side effects induced by the therapeutic agents used, as well as disease-associated factors that can negatively affect QOL [4].

Taking into consideration the chronic often incurable nature of the disease and the high risk of recurrence, cancer has a significant effect on QOL. The diagnosis by itself can cause different psychological problems like anxiety, fear, anger, sadness, and depression which compromise QOL [5]. Though gynaecologic cancer constitutes a large proportion of female cancers in Ethiopia, HRQOL of gynaecologic cancer patients is not assessed yet. Therefore this study aimed to investigate HRQOL of gynaecologic cancer patients using the Amharic version of EORTC QLQ-C30 attending at TASH, Addis Ababa, Ethiopia.

\section{Methods}

\section{Study setting}

This study was conducted at the oncology center of Tikur Anbesa Specialized Hospital, Addis Ababa, Ethiopia. The hospital is a teaching hospital of Addis Ababa University and a major referral center from all corners of the country especially for cancer patients. The hospital has a variety of specialty and sub-specialty training in varies fields of study including gynaecology/ obstetrics, surgery and oncology. The hospital owned the only oncology center in the nation providing radiation therapy during the study period. The oncology center has about 500 adult and pediatric cases with hematologic malignancies every year. The most common adult cancers were cervical, breast, sarcomas, head and neck, and colorectal cancers.

\section{Study design and period}

This study employed a facility based cross-sectional study on gynaecologic cancer patients attending TASH between January 1 st to June $30^{\text {th }} 2014$. The study team approached a total of 153 gynaecologic cancer patients. However 13 patients were excluded from the study because they refused to provide information on the variables of interest of this study after they gave their consent participate.

\section{Quality of life assessment tool}

The quality of life was assessed by Amharic version of EORTC QOL-C30 [6]. The study group evaluated the reliability and validity of the questionnaire and found the reliability and validity of the tool was in acceptable range despite its few limitations [7].

\section{Study subjects}

We only included adult gynaecologic cancer patients' age $\geq 18$ years and who were taking oncologic treatment for the first time. Those adult gynaecologic cancer patients who had received oncological treatment previously were excluded from study because the treatment by itself can compromise QOL significantly. We also excluded those patients with psychiatric disorders, communication disorders, severe other medical illnesses or diagnosed with coexisting malignancies and HIV positive patients because all this health problems compromise the quality of life which could confound the impact of the cancer on quality of life.

\section{Statistical analysis}

Before the data were entered to SPSS version 20 for windows, it was checked for completeness, inconsistencies and cleaned by the study team. The team also did data cleaning on the entered data and double-check its consistency with the paper questionnaire. The raw scores were transformed to scores ranging from 0 to 100. Linear transformation to 0-100 to obtain the score $\mathrm{S}$, have been done according to the formula given by EORTC [6].

Descriptive statistics (frequencies, mean, and standard deviation) were used to summarize the data. Bivariate analyses (independent $t$ test and one way ANOVA) were used to determine the association between the QOL and patient socio-demographic and clinical characteristics. A $p$ value of $<0.05$ was used to declare a statistically significant association.

\section{Results}

Socio-demographic and clinical characteristics of patients In this study, the study team approached a total of 153 study subjects; however only 140 gynaecologic cancer patients who had completed data included in the 
analysis. The socio-demographic characteristics of the gynaecologic cancer patients included in this study were depicted in our published article of the validity section of this study [7].

Cervical cancer represents the most frequent cancer type in the study, diagnosed in $77.2 \%$ of patients and it was followed by ovarian and endometrial cancer $(15 \%$ and $5 \%$ respectively). The majority of patients 55(47\%) were either at stage 3 or 4 , and followed by stage two 49(41.9\%) (Table 1$)$.

\section{Quality of life scores}

The mean Global Health Status (global quality of life) score was $40.95(\mathrm{SD} \pm 24.35)$ and of the functional scales, social function 42.26 ( $\mathrm{SD} \pm 32.08$ ), was the most affected domain followed by role function $50.12(\mathrm{SD} \pm 35.11$ ) whereas cognitive function is least affected (mean = 88.21, SD \pm 18.49 ). The highest score on the symptom scale was for financial difficulties (mean $=64.76$, $\mathrm{SD} \pm 32.43$ ) followed by pain (mean $=55.12, \mathrm{SD} \pm 29.64$ ) and fatigue (mean $=53.97, \mathrm{SD} \pm 28.54$ ) while the lowest score was for diarrhea $($ mean $=1.19, \mathrm{SD} \pm 7.38)$ (Table 2$)$.

\section{Association of quality of life scores with socio- demographic and clinical characteristics}

As stage increases there is a statistically significant reduction in GHS $(p=0.005)$ and in all functional scale domains [physical function $(p=0.002)$, role function $(p=0.032)$ and cognitive function $(P<0.001)]$ except social function $(p=0.065)$ and emotional function $(p=$ 0.149) (Table 3). Advancement in stage of the disease has also affected significantly the symptom scale domains such

Table 1 Clinical characteristics of gynaecological cancer patients attending treatment at TASH, Addis Ababa, Ethiopia, 2014

\begin{tabular}{llll}
\hline Clinical characteristics & & Frequency & Percent \\
\hline Cancer type & Cervical & 108 & 77.2 \\
& Ovarian & 21 & 15.0 \\
& Endometrial & 7 & 5.0 \\
& Others(vaginal, vulvar) & 4 & 2.8 \\
Stage of the diseases & 1 & 12 & 10.3 \\
$(n=117)$ & 2 & 49 & 41.9 \\
& 3 and 4 & 55 & 47.0 \\
Treatment type & Radiation & 84 & 60.0 \\
& Operation & 56 & 40.0 \\
Payment type & Paying & 103 & 73.6 \\
& Free & 37 & 26.4 \\
Care givers relation & Children & 96 & 68.6 \\
& Spouse & 25 & 17.9 \\
& Sibling & 10 & 7.1 \\
& Parents & 9 & 6.4 \\
\hline
\end{tabular}

Table 2 Mean and standard deviation of EORTC- QLQ C-30 components for gynaecological cancer patients at TASH, Addis Ababa, Ethiopia, 2014

\begin{tabular}{llll}
\hline EORTC- QLQ C-30 components & Items & Mean & Std. Deviation \\
\hline GHS & 29,30 & 40.95 & 24.35
\end{tabular}

Functional scale

\begin{tabular}{llll} 
Physical function & $1-5$ & 65.24 & 22.59 \\
Role function & 6,7 & 50.12 & 35.11 \\
Emotional function & $21-24$ & 55.48 & 30.32 \\
Cognitive function & 20,25 & 88.21 & 18.49 \\
Social function & 26,27 & 42.26 & 32.08 \\
Symptom scale & & & \\
Fatigue & $10,12,18$ & 53.97 & 28.54 \\
Nausea and Vomiting & 14,15 & 8.21 & 17.92 \\
Pain & 9,19 & 55.12 & 29.65 \\
Dyspnea & 8 & 6.67 & 17.97 \\
Insomnia & 11 & 36.19 & 33.57 \\
Appetite loss & 13 & 44.05 & 36.71 \\
Constipation & 16 & 44.29 & 39.06 \\
Diarrhea & 17 & 1.19 & 7.38 \\
Financial difficulties & 28 & 64.76 & 32.43 \\
\hline
\end{tabular}

as fatigue $(p<0.001)$, pain $(p<0.001)$, insomnia $(p=0.002)$, and constipation $(p<0.001)$ and appetite loss $(p=0.013)$. But it was not significantly associated with financial difficulties $(p=0.32)$, nausea/vomiting $(p=0.35)$ and diarrhea $(p=$ 0.87) (Table 4).

Patients coming from outside Addis Ababa had significantly lower score for social function compared to those who were from Addis Ababa $(p=0.03)$ (Table 3). Insomnia $(p=0.02)$, and financial difficulties $(p=0.036)$ were significantly higher in those patients who were coming from outside Addis Ababa (Table 4). Patients who never went to school had significantly lower score in GHS $(p=0.013)$, physical function $(p=0.036)$, role function $(p=0.008)$ and social function $(p=0.002)$ (Table 4$)$. Those patients who never went to school had significantly higher score for symptom scales such as fatigue $(p=0.001)$, pain $(p<0.001)$, dyspnea $(p=0.001)$, insomnia $(p=$ $0.002)$, financial difficulties $(p=0.015)$, and constipation $(p<0.001)$ (Table 4$)$.

Marital status was significantly associated with emotional function and appetite loss. This study found that those married patients had significantly higher score on emotional function than their unmarried counter parts $(p=0.037)$. Appetite loss score was significantly higher among those married patients $(p=0.028)$ than their unmarried counter parts. The score for role function among patients who were paying was significantly higher as compared to patients who were free of charge $(p=0.021)$. Financial difficulties $(p=0.046)$, 


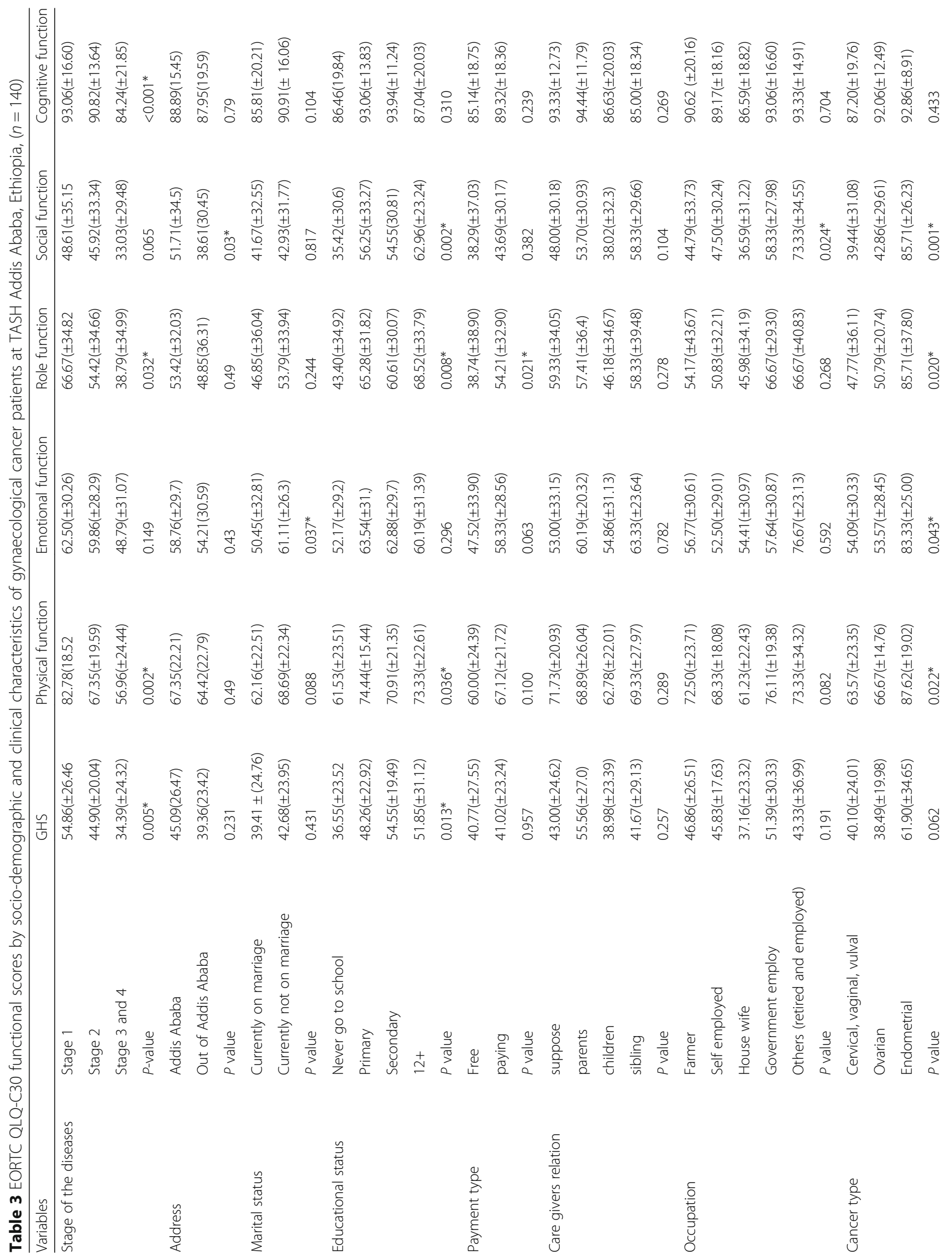




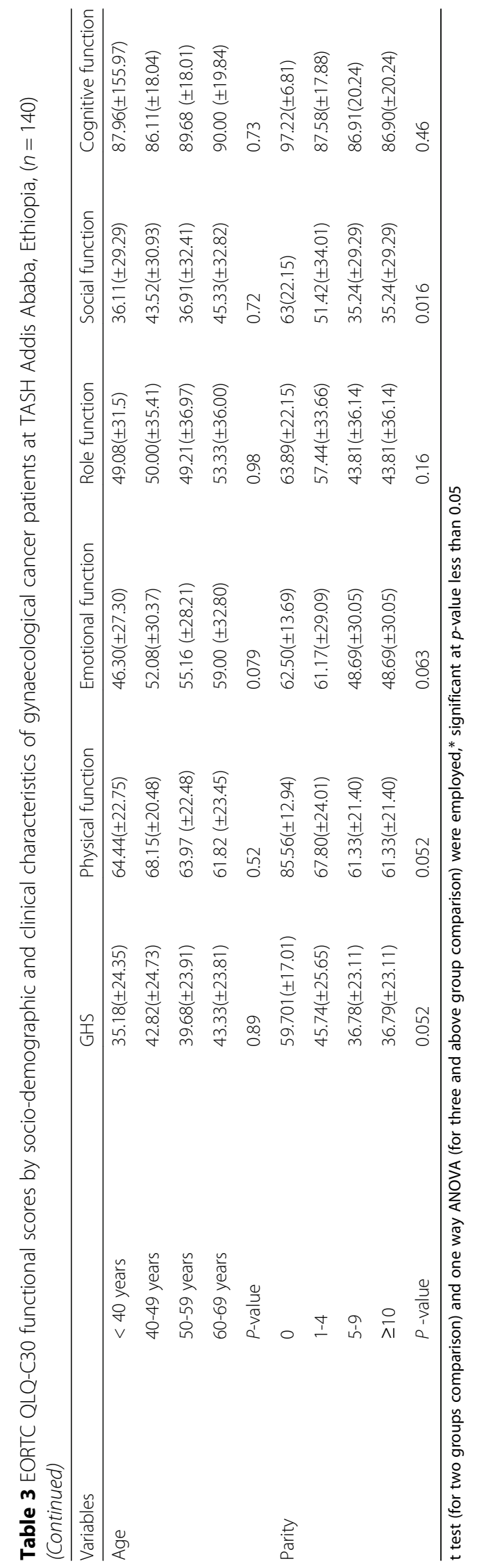




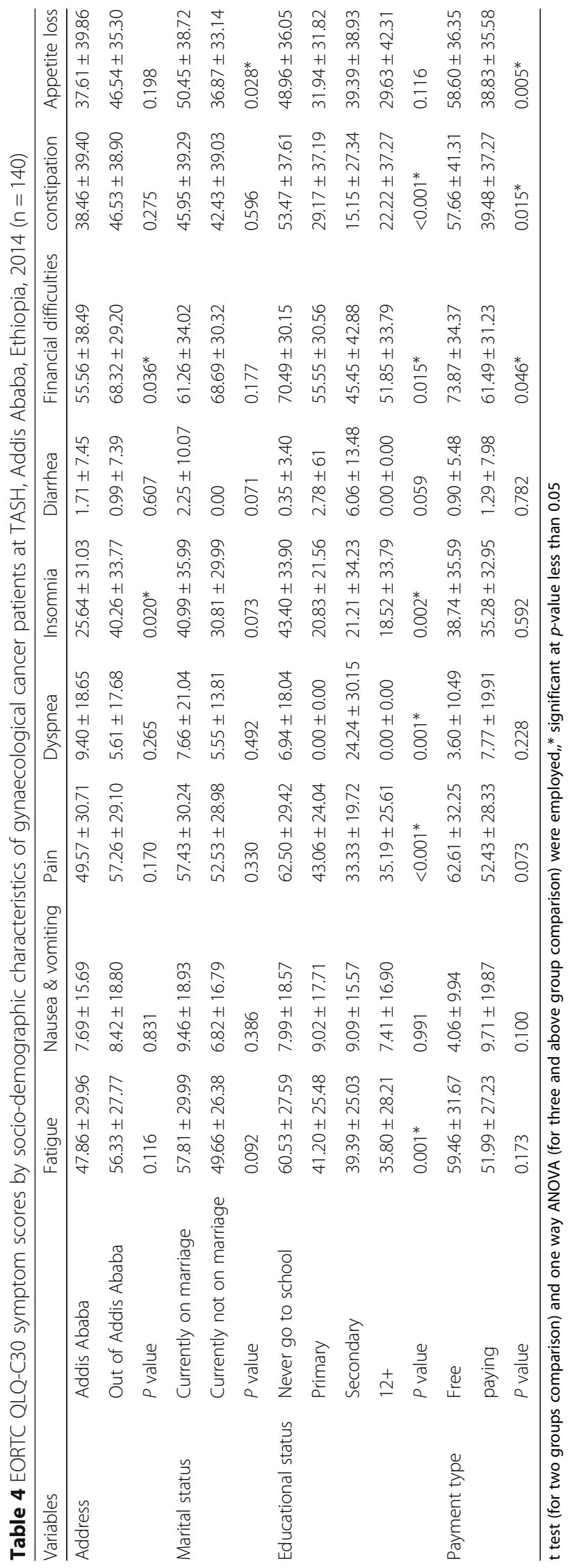


constipation $(p=0.015)$ and appetite loss $(p=0.005)$ were also scored low among paying group (Table $3 \& 4$ ). Parity was significantly associated with social function $(p=$ $0.016)$, fatigue $(p=0.015)$, pain $(p=0.024)$, financial difficulties $(p=0.003)$, appetite loss $(p=0.003)$. This study found that EORTC-Q30 functional scale domains varied significantly depending on the gynecologic cancer type. Endometrial cancer was associated with a better score in GHS; in all functional scale $(P$ value $\leq 0.05)$ except cognitive function (Table 3 ) and in all symptom scales domains $(p \leq 0.05)$ except constipation, diarrhea and insomnia (Table 5). Cervical cancer patients had significantly worse score in fatigue and pain $(p \leq 0.05)$. As shown on Table 5, having ovarian cancer was associated with a higher score in dyspnea and nausea/vomiting than other types of cancers $(\mathrm{p} \leq 0.05)$. Endometrial cancer has a better score in fatigue, pain and financial difficulties as compared to the other cancer types $(\mathrm{Pp} \leq$ 0.05) (Table 5).

\section{Discussion}

Social function was the most affected while cognitive function was the least affected domain among the functional scale in gynaecological cancer patients in Ethiopia. Among symptom scale, financial difficulty was the worst affected QOL dimension. Studies conducted in other developing countries, Tanzania and Indonesia showed that financial difficulty is one of the most affected domain among the symptom scale $[8,9]$. We found that pain and fatigue were the second and the third highly scored domains whereas a Turkish and Malaysian studies showed that either of the two are the most affected domains $[10,11,12]$. This may be due to the fact of inaccessibility of the oncology service and lack of health insurance in the Ethiopian settings. This makes patients to travel over a long distance to get oncologic service which could be the cause of fatigue and pain. The mean score for GHS in this study was 40.95 which is a little lower than the Tanzanian report (50.5) performed amongst all types of cancer [8]. This difference could be attributed to the difference in socio-demographic characteristics, cancer type and health services access between Ethiopia and Tanzania.

This study found that among the socio demographic characteristics, marital status was significantly associated with emotional function and appetite loss. However, elsewhere marital status has shown to have inconsistent association with quality of life. In one Iranian study [13] marital status was found not correlated in any of the scores where as a study done in Turkey $[10,14]$ showed married participants to have a low score in emotional function in contrary to our finding.

Patients from outside Addis Ababa have a lower score of social function and a higher score of insomnia and financial difficulties. Traveling from home to Addis Ababa, where the service is available creates additional expense for traveling; the need for somebody to accompany them and the need to stay in a new environment during the treatment period. This could affect their social function, insomnia and financial difficulties. Additional evidence that showed financial difficulties can compromise other quality of life domain is that those patients who were being treated free of charge because they are poor have low score of role function and high score in financial difficulty, high score in constipation and high score in appetite loss.

Educational status has also shown a significant correlation with health related quality of life components. Those patients who had never gone to school scored lower in GHS, physical function, and social function and had higher score in fatigue, pain, dyspnea, financial difficulties and constipations. A study done in Turkey showed education status was associated with physical function and pain in the same way as ours but in contrary to ours with fatigue [10]. This can be justified by the fact that a lower level of education is associated with poor health seeking behavior. This study also found that those patients who are house wife had a lower score in social function. There was a similar finding from Sudan which showed higher QOL of scores for patients when they are employed in medium skill/high skill occupation [15].

The EORTC-Q30 scores varied based on cancer type within the broad category of Gynaecologic cancer. Endometrial cancer patients scored better in most of the functional and symptom scale domains. This can be explained by the early presentation of the endometrial cancers as compared to the others. The Turkish study has also shown endometrial cancer to be favored in role function, social function, fatigue and financial difficulties [10]. Stage of the disease and the intended type of treatment were associated with quality of life of cervical cancer patients. Advanced stage of diseases and radiation treatment found associated with the worst side [7]. A study done in Texas has described cervical cancer survivors treated with radiotherapy to report more QOL impairments than survivors treated with other approaches [16].

\section{Limitation of the study}

This study had the following major limitations. This study did not employed multivariable regression analysis to control the confounders because we have many scores used as indicator of quality of life. The study was conducted using Amharic version of EORTC QLQ-C30 despite the fact that all study participants were not Amharic native speakers. EORTC QLQ-C30 questionnaire was also not tested for its responsiveness and test-retest reliability. 


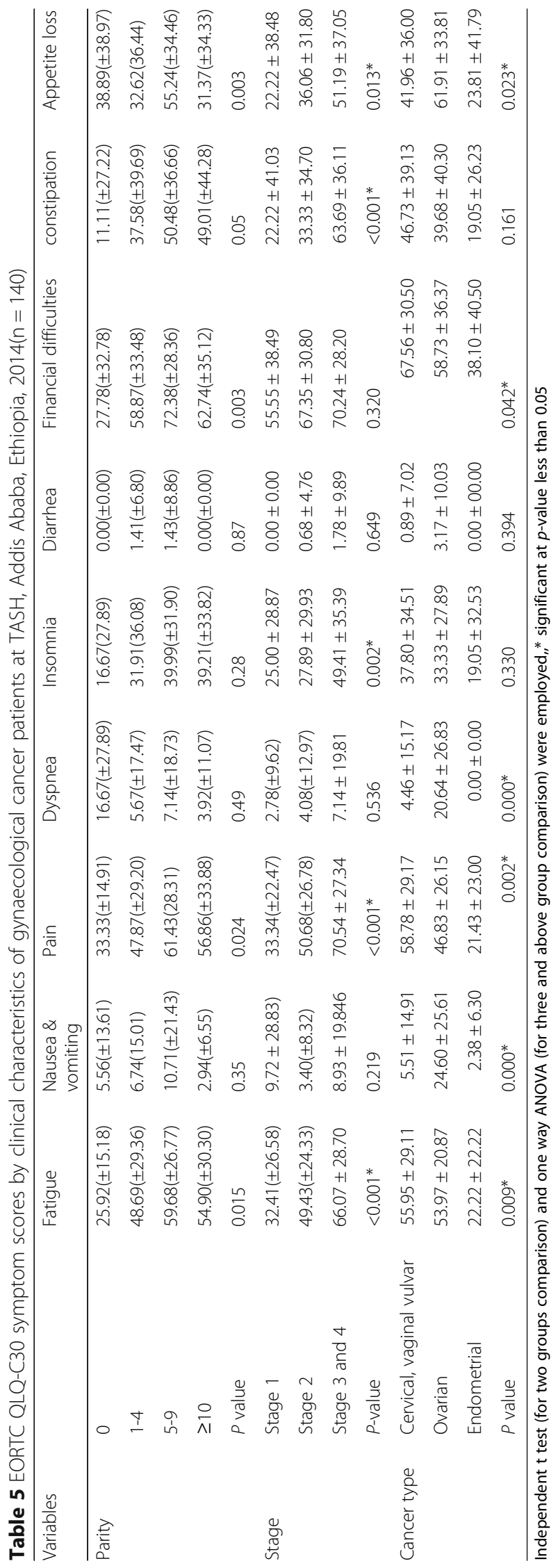




\section{Conclusion}

GHS, social function, financial difficulties, pain and fatigue were the most affected however cognitive function and diarrhea were less affected components of HRQOL of gynaecological cancer patients. Place of residence, educational status, marital status, payment type, cancer type and stage of the disease were some of the different socio demographic and disease related factors were associated with health related quality of life.

\section{Abbreviations}

ANOVA: Analysis of Variance; EORTC QLQ- C30: European Organization for Research and Treatment of Cancer Quality of life questionnaire core-30; GHS: Global Health Function; HRQOL: Health Related Quality of Life; QOL: Quality of life; TASH: Tikur Anebesa Specialized Hospital; WHO: World Health Organization

\section{Acknowledgements}

The authors would like to thank Addis Ababa University for funding this study. The funders had no role in study design, data collection and analysis, decision to publish, or preparation of the manuscript. We also thank the study subjects to be participated in this study. We forward our greatest gratitude to EORTC who allowed us to use the questionnaire. Moreover Dr. Dereje $\mathrm{H}$ who gave us his advice and comments throughout of the study also deserve our heartfelt thanks.

\section{Funding}

This study was funded by school of Medicine, Addis Ababa University, Ethiopia. The funders had no role in study design, data collection and analysis, decision to publish, or preparation of the manuscript.

\section{Availability of data and materials}

Data can be obtained from the first author of this manuscript on reasonable request.

\section{Authors' contributions}

BA conceived and designed the study, performed analysis, interpretation of data and drafted the manuscript. SN assisted with the design, conception, and interpretation of data and critically reviewed the manuscript. LY assisted with the design, conception, and interpretation of data and critically reviewed the manuscript. WT assisted with the design, conception, and interpretation of data and critically reviewed the manuscript. DH assisted with the design, conception, analysis, and interpretation of data and critically reviewed the manuscript. All authors read and approved the final manuscript.

\section{Ethics approval and consent to participate}

The study was conducted after getting ethical clearance from Addis Ababa University, school of medicine, Department of gynaecology and obstetrics research committee (ch/SoM/112/13). Permission was requested from the EORTC to use the EORTC QLQ- C30 questionnaire. Patients were informed about the purpose of the study and the importance of their participation to confirm willingness for participation. Patients were assured that the completed questionnaires will not be stored in the patient's clinical notes and will remain confidential. Finally, a written consent was taken from the patients before the interview.

\section{Consent for publication}

Not applicable.

\section{Competing interests}

The authors declare that they have no competing interests.

\section{Author details}

'Department of Gynecology \& Obstetrics, College of Medicine and Health Sciences, University of Gondar, Gondar, Ethiopia. ${ }^{2}$ Department of Gynecology \&Obstetric, School of Medicine, College of Health sciences, Addis Ababa University, Addis Ababa, Ethiopia. ${ }^{3}$ Department of Oncology, School of Medicine, College of Health sciences, Addis Ababa University, Addis Ababa, Ethiopia. ${ }^{4}$ Department of Reproductive Health and Health Service Management, School of Public Health, College of Health sciences, Addis Ababa University, Addis Ababa, Ethiopia.

Received: 12 January 2017 Accepted: 28 December 2017 Published online: 05 January 2018

\section{References}

1. Arriba LN, Fader AN, Frasure HE, VonGruenigen VE: A review of issues surrounding quality of life among women with ovarian cancer. Gynecol Oncol. 2010 doi: 101016/j yayno201005014.

2. Bowling A: Health-related quality of LIFE: a discussion of the concept, its use and measurement background: the 'QUALITY OF LIFE' 'Presented to the adapting to change Core course. 1999.

3. Tabano M, Condosta D, Coons M. Symptoms affecting quality of life in women with Gynaecologic cancer. Semin Ontology Nurs. 2002;18(3):223-30.

4. Wenzel L, Vergote I, Cella D. Quality of life in patients receiving treatment for gynecologic malignancies: special considerations for patient care. Int J Gynaecol Obstet. 2003;83(1):211-29.

5. Pignata S, Ballatori E, Favalli G, Scambia G. Quality of life: gynaecological cancers. Ann Oncol. 2001;12(3):S37-42.

6. Scott NW, Fayers PM, Aaronson NK, Bottomley NK, Groenvold M, Gundy C: On behalf of the EORTC quality of life group ,EORTC QLQ-C30 reference values, 2008.

7. Ayana BA, Negash S, Yusuf L, Tigeneh W, Haile D. Reliability and validity of Amharic version of EORTC QLQ-C 30 questionnaire among gynecological cancer patients in Ethiopia. PLoS One. 2016;11(6):e0157359.

8. Masika GM, Wettergren L, Kohi TW, von Essen L. Health-related quality of life and needs of care and support of adult Tanzanians with cancer: a mixedmethods study. Health Qual Life Outcomes. 2012;10:133.

9. Pradjatmo H, Nisman WA, Fatmawati Y. Quality of life of cervical cancer patient with support from nuclear family and extended family in Dr. Sardjito general hospital, Yogyakarta Indonesia: a comparative study. Int J Res Med Sci. 2017;5(8):3554-9.

10. Goker A, Guvenal T, Yanikkerem E, Turhan A, Koyuncu FM. Quality of life in women with Gynaecologic cancer in Turkey. Asian Pacific J Cancer Prev. 2011;12:3121-8.

11. Farooqui $\mathrm{M}$, et al. Cross sectional assessment of health related quality of life (HRQoL) among patients with cancer in Malaysia. Asian Pacific J Cancer Prev. 2013:14(5):3017-21.

12. Theobald DE. Cancer pain, fatigue, distress, and insomnia in cancer patients. Clin Cornerstone. 2004;6:S15-21.

13. Silpakit C, Sirilerttrakul S, Jirajarus M, Sirisinha T, Sirachainan E, Ratanatharathorn V. The European Organization for Research and Treatment of cancer quality of life questionnaire (EORTC QLQ-C30): validation study of the Thai version. Qual Life Res. 2006;15:167-72.

14. Hoopman R, Muller MJ, Terwee CB, Aaronson NK. Translation andvalidation of the EORTC QLQ-C30 for use among Turkish and Moroccan ethnic minority cancer patients in the Netherlands. Eur J Cancer. 2006:42:1839-47.

15. Awadalla AW, Ohaeri JU, Gholoum A, Khalid AO, Hamad HM, Jacob A. Factors associated with quality of life of outpatients with breast cancer and gynaecologic cancers and their family caregivers: a controlled study. BMC Cancer. 2007;7(1):102.

16. Frumovitz M, Sun CC, Schover LR, Munsell MF, Jhingran A, Wharton JT, Eifel P, Bevers TB, Levenback CF, Gershenson DM, et al. Quality of life and sexual functioning in cervical cancer survivors. J Clin Oncol. 2005;23(30):7428-36.

\section{Publisher's Note}

Springer Nature remains neutral with regard to jurisdictional claims in published maps and institutional affiliations. 